\title{
Ising spins on discrete Regge lattices
}

\author{
E. Bittner ${ }^{\mathrm{a}, \mathrm{b}}$, W. Janke ${ }^{\mathrm{b}, *}$, H. Markum ${ }^{\mathrm{a}}$, J. Riedler ${ }^{\mathrm{a}}$ \\ ${ }^{a}$ Institut für Kernphysik, Technische Universität Wien, Wiedner Hauptstraße 8-10, \\ A-1040 Vienna, Austria \\ ${ }^{\mathrm{b}}$ Institut für Theoretische Physik, Universität Leipzig, Augustusplatz 10/11, D-04109 Leipzig, Germany
}

Received 6 October 1999

\begin{abstract}
We study an Ising spin system coupled to the fluctuating two-dimensional geometry of the discrete Regge model and compare with the results of the standard Regge calculus. Particular emphasis is placed on the phase transition of the spin system and the associated critical exponents. The results are obtained from finite-size scaling analyses of extensive Monte Carlo simulations. (c) 2000 Elsevier Science B.V. All rights reserved.
\end{abstract}

\section{Introduction}

Spin systems coupled to fluctuating two-dimensional manifolds are studied as a simple example for matter fields coupled to Euclidean quantum gravity. From the view of statistical mechanics one investigates the properties of a special kind of annealed disorder, interpreted physically as fluctuating "space-time". A particularly sensitive probe of the properties of this coupling are phase transitions of the spin system and the associated critical exponents. In the case of the two-dimensional Ising spin system, conformal field theory arguments predict that the annealed disorder caused by the fluctuating geometry modifies the critical exponents from the flat-space Onsager values $\alpha=0, \beta=\frac{1}{8}$, $\gamma=\frac{7}{4}$, and $v=1$ to the KPZ [1] values $\alpha=-1, \beta=\frac{1}{2}, \gamma=2$, and $D v=3$, where $D$ is the internal fractal dimension of the two-dimensional manifold. While these predictions could be confirmed numerically within the dynamically triangulated random surface (DTRS) approach [2-5], it was found that the Ising model on lattices of toroidal [6,7] as well as spherical [8] topology stays in the flat-space Onsager universality class when coupled to gravity via Regge calculus [9].

\footnotetext{
* Corresponding author. Fax: +49-341-97-32-747.

E-mail address: wolfhard.janke@itp.uni-leipzig.de (W. Janke)
} 
The latter results came somewhat unexpected, because it was generally believed that both approaches should describe the same model in the continuum limit. To explain this discrepancy the prevailing opinion is that the path-integral measure in the Regge approach should be non-local [10-12]. A numerical study with a non-local measure is, however, an extremely time demanding enterprise from a computational point of view and one therefore seeks for suitable approximations. One such candidate is the discrete Regge model [13-16] where the squared link lengths of the triangulated manifold are constrained to take on only two different values. This could be the desired simplified formulation for further numerical investigations employing non-local pathintegral measures in the Regge approach. In this paper we study an Ising spin system coupled to the two-dimensional discrete Regge model using Monte Carlo computer simulations and compare the results of our simulations with those of the standard Regge calculus [7].

The rest of the paper is organized as follows. In Section 2 we introduce the discrete Regge model and give some details of the Monte Carlo simulation. The results are presented in Section 3, and concluding remarks can be found in Section 4.

\section{Model and simulation}

One of the oldest methods to study general relativity numerically was suggested by Regge [9]. He applies a simplicial approximation to a manifold, where the underlying lattice has fixed connectivities (in contrast to the DTRS approach [2-5]), and takes the squared link lengths $q_{i j}$ as gravitational degrees of freedom. The discrete Regge model approach [13-16] employed in this work is structurally and computationally much simpler than the standard Regge calculus. All the squared link lengths are restricted to take on only the two values

$$
q_{i j}=1+\varepsilon \sigma_{i j}, \quad 0 \leqslant \varepsilon<\frac{3}{5}, \quad \sigma_{i j} \in Z_{2} .
$$

The parameter $\varepsilon$ is a real number chosen such that the Euclidean triangle inequalities are always fulfilled. In the actual simulations we choose $\varepsilon=\frac{1}{2}$. Hence, with $N_{1}$ denoting the total number of links, all $2^{N_{1}}$ possible configurations are allowed. This is quite different from the standard Regge calculus where many potential updates of the link lengths either violate the triangle inequality or the manifold property. Furthermore, the $Z_{2}$ restriction may provide a natural measure attributing the same a priori weight to all $2^{N_{1}}$ configurations.

We use the usual transcription of continuum quantities such as the metric $g$ and the scalar curvature $R$ into the Regge approach, namely,

$$
\int \mathrm{d}^{2} x \sqrt{g(x)} \rightarrow \sum_{i} A_{i},
$$




$$
\begin{gathered}
\int \mathrm{d}^{2} x \sqrt{g(x)} R(x) \rightarrow 2 \sum_{i} \delta_{i}, \\
\int \mathrm{d}^{2} x \sqrt{g(x)} R^{2}(x) \rightarrow 4 \sum_{i} \frac{\delta_{i}^{2}}{A_{i}}
\end{gathered}
$$

with $\delta_{i}$ denoting the deficit angle at the vertex $i$ defined as

$$
\delta_{i}=2 \pi-\sum_{\text {all triangles } t \text { sharing } i} \theta_{i}(t),
$$

where $\theta_{i}(t)$ is the dihedral angle associated with the triangle $t$. The area $A_{i}$ is taken to be the barycentric area associated with the site $i$,

$$
A_{i}=\sum_{t \supset i} \frac{1}{3} A_{t}
$$

where $A_{t}$ denotes the area of the triangle $t$. We simulate the partition function

$$
Z=\sum_{\{s\}} \int \mathrm{D} \mu(q) \exp [-I(q)-K E(q, s)],
$$

where $I(q)$ is the standard gravitational action with cosmological constant $\lambda$,

$$
I(q)=\sum_{i}\left(\lambda A_{i}+a \frac{\delta_{i}^{2}}{A_{i}}\right)
$$

and

$$
E(q, s)=\frac{1}{2} \sum_{\langle i j\rangle} A_{i j} \frac{\left(s_{i}-s_{j}\right)^{2}}{q_{i j}}
$$

is the energy of Ising spins $s_{i} \in Z_{2}$ located at the vertices $i$ of the lattice. Here, the volume $A_{i j}$ associated with a link $\langle i j\rangle$ is defined as

$$
A_{i j}=\sum_{t \supset\langle i j\rangle} \frac{1}{3} A_{t} .
$$

In two dimensions the integral over the scalar curvature is a topological invariant due to the Gauss-Bonnet theorem. Its simplicial analogue reads

$$
\sum_{i \supset K} \delta_{i}=2 \pi \chi(\mathscr{M}),
$$

where $\chi=2(1-g)$ is the Euler characteristic of the manifold $\mathscr{M}$ expressed by the number of handles $g$ in $\mathscr{M}$. For a simplicial complex $K$ the Euler characteristic can be computed as

$$
N_{0}-N_{1}+N_{2}=2(1-g) \text {, }
$$

where $N_{0}, N_{1}$, and $N_{2}$ denote the number of sites, links, and triangles, respectively. In higher than two dimensions an $R^{2}$ interaction term is sometimes added to guarantee 
the boundedness of the gravitational action from below. This is not necessary in two dimensions but we include such a term to investigate its influence on the continuum limit. We use the same path-integral measure $D \mu(q)=\prod_{l}\left(d q_{l} / q_{l}^{m}\right) \mathscr{F}\left(q_{l}\right)$ as in the pure gravity simulations [16], with the integration in (2.7) replaced by a summation over the $Z_{2}$ degrees of freedom $\sigma_{l} \equiv \sigma_{i j}$. The constraint $\mathscr{F}$, which assures that only Euclidean configurations of links can contribute, is actually superfluous in the discrete Regge model where this is always satisfied by construction. Here, it is only included for completeness and for comparison with standard Regge calculus where it is an important part of the measure. The real parameter $m$ allows to choose a particular measure. For reasons discussed in detail in Ref. [16] we take $m=-2 \lambda c_{1} / M$ with $c_{1}=[2 \sqrt{3}+\sqrt{(1-\varepsilon)(3+5 \varepsilon)}-\sqrt{(1+\varepsilon)(3-5 \varepsilon)}] / 32$ and $M=\frac{1}{2} \ln [(1+\varepsilon) /(1-\varepsilon)]$ which, for $\varepsilon=\frac{1}{2}$, amounts to $m \approx-0.3 \lambda$.

We simulated the gravitational action using the standard single-hit Metropolis algorithm. To prevent critical slowing down $[17,18]$ near the phase transition we employed the single-cluster (Wolff) algorithm [19,20] for the Ising spins. Between measurements we performed $n=10$ Monte Carlo steps consisting of one lattice sweep to update the squared link lengths $q_{i j}=1+\sigma_{i j} / 2$ followed by two single-cluster flips to update the spins $s_{i}$.

The primary simulations are done for cosmological constant $\lambda=1.0$ and squared curvature coupling $a=0.001$. In the present study the total area $A=\sum_{i} A_{i}$ can thus fluctuate around its mean value while in the investigations of standard Regge calculus in Ref. [7] the total area was kept fixed. The lattice topology is given by triangulated tori of size $N_{0}=L^{2}$ with $L=8,16,24,32,48,64,96,128$, and 256. From short test runs we estimated the location of the phase transition and set the spin coupling $K_{0}=1.040 \approx K_{c}$ in the long runs. Analogous simulations are performed with $a=0$ on the same lattice sizes except the largest. The difference between the model defined by (2.7) and the Ising model on a static triangular lattice is that the spins are coupled by geometric weight factors $w_{i j}=A_{i j} / q_{i j}$ which can fluctuate around the static value $w_{i j}^{0}=\sqrt{3} / 6$.

For every run we record the time series of the energy density $e=E / N_{0}$ and the magnetization density $m=\sum_{i} A_{i} s_{i} / N_{0}$. After an initial equilibration time we take about 50000 measurements for each lattice size. Analyzing the time series we find integrated autocorrelation times for the energy and the magnetization in the range of unity for all lattice sizes. To obtain results for the various observables $\mathcal{O}$ at values of the coupling $K$ in an interval around the simulation point $K_{0}$, we apply the reweighting method [21]. Since we record the time series, this amounts to computing

$$
\left.\langle\mathcal{O}\rangle\right|_{K}=\frac{\left.\left\langle\mathcal{O} \mathrm{e}^{-\Delta K E}\right\rangle\right|_{K_{0}}}{\left.\left\langle\mathrm{e}^{-\Delta K E}\right\rangle\right|_{K_{0}}}
$$

with $\Delta K=K-K_{0}$. The statistical errors are obtained by the standard Jack-knife method using 50 blocks.

With the help of the time series we compute the specific heat

$$
C(K)=K^{2} N_{0}\left(\left\langle e^{2}\right\rangle-\langle e\rangle^{2}\right),
$$


Table 1

Fit results for $1 / v$ and $a=0.001$. The average is computed by weighting each entry with its inverse squared error. For the fits at our best estimate of $K_{c}=$ 1.0393(3) the uncertainty due to the error in $K_{c}$ is indicated by the numbers in square brackets

\begin{tabular}{llll}
\hline Fit-type & $N$ & $1 / v$ & $Q$ \\
\hline $\mathrm{d} U / \mathrm{d} K$ at $K_{\max }^{C}$ & 7 & $0.949(86)$ & 0.96 \\
$\mathrm{~d} \ln \langle|m|\rangle / \mathrm{d} K$ at $K_{\inf }^{\ln \langle|m|\rangle}$ & 7 & $0.987(33)$ & 0.71 \\
$\mathrm{~d} \ln \left\langle m^{2}\right\rangle / \mathrm{d} K$ at $K_{\inf }^{\ln \left\langle m^{2}\right\rangle}$ & 7 & $0.995(32)$ & 0.66 \\
Weighted average & & $0.988(22)$ & \\
& 7 & $0.986(9)[30]$ & 0.51 \\
$\mathrm{~d} U / \mathrm{d} K$ at $K_{c}$ & 7 & $1.007(6)[20]$ & 0.24 \\
$\mathrm{~d} \ln \langle|m|\rangle / \mathrm{d} K$ at $K_{c}$ & 7 & $1.011(5)[18]$ & 0.11 \\
$\mathrm{~d} \ln \left\langle m^{2}\right\rangle / \mathrm{d} K$ at $K_{c}$ & & $1.006(13)$ & \\
Weighted average & & & \\
Overall average & & $1.001(11)$ & \\
\hline
\end{tabular}

the (finite lattice) susceptibility,

$$
\chi(K)=N_{0}\left(\left\langle m^{2}\right\rangle-\langle|m|\rangle^{2}\right)
$$

and various derivatives of the magnetization, $\mathrm{d}\langle|m|\rangle / \mathrm{d} K, \mathrm{~d} \ln \langle|m|\rangle / \mathrm{d} K$, and $\mathrm{d} \ln \left\langle m^{2}\right\rangle / \mathrm{d} K$. All these quantities exhibit in the infinite-volume limit singularities at $K_{c}$ which are shifted and rounded in finite systems. We further analyze the Binder parameter,

$$
U_{L}(K)=1-\frac{1}{3} \frac{\left\langle m^{4}\right\rangle}{\left\langle m^{2}\right\rangle^{2}} .
$$

It is well known that the $U_{L}(K)$ curves for different lattice sizes $L$ cross around $\left(K_{c}, U^{*}\right)$ with slopes $\propto L^{1 / v}$, apart from confluent corrections explaining small systematic deviations. This allows an almost unbiased estimate of the critical coupling $K_{c}$, the critical correlation length exponent $v$, and the renormalized charge $U^{*}$.

\section{Results}

Applying the reweighting technique we first determine the maxima of $C, \chi, \mathrm{d}\langle|m|\rangle / \mathrm{d} K$, $\mathrm{d} \ln \langle|m|\rangle / \mathrm{d} K$, and $\mathrm{d} \ln \left\langle m^{2}\right\rangle / \mathrm{d} K$. The locations of the maxima provide us with five sequences of pseudo-transition points $K_{\max }(L)$ for which the scaling variable $x=$ $\left(K_{\max }(L)-K_{c}\right) L^{1 / v}$ should be constant. Using this fact we then have several possibilities to extract the critical exponent $v$ from (linear) least-squares fits of the finite-size scaling (FSS) ansatz $\mathrm{d} U_{L} / \mathrm{d} K \cong L^{1 / v} f_{0}(x)$ or $\mathrm{d} \ln \left\langle|m|^{p}\right\rangle / \mathrm{d} K \cong L^{1 / v} f_{p}(x)$ to the data at the various $K_{\max }(L)$ sequences. Note that by replacing $L^{1 / v}$ by $N_{0}^{1 / D v}$ we trivially get the same estimates for $1 / v$ and $2 / D v$ which would account for a possibly non-trivial internal fractal dimension as in the DTRS approach. The exponents $1 / v$ resulting from fits using the data for the $N$ largest lattice sizes are collected in Tables 1 and 2. Here, $Q$ denotes the standard goodness-of-fit parameter. For the simulations at $a=0.001$ all exponent estimates and consequently also their weighted average $1 / v=0.988(22)$ are in 
Table 2

Fit results for $1 / v$ and $a=0$ using $K_{c}=1.0395(3)$. The averages and statistical errors are computed as in Table 1

\begin{tabular}{llll}
\hline Fit-type & $N$ & $1 / v$ & $Q$ \\
\hline $\mathrm{d} U / \mathrm{d} K$ at $K_{\max }^{C}$ & 7 & $0.964(49)$ & 0.63 \\
$\mathrm{~d} \ln \langle|m|\rangle / \mathrm{d} K$ at $K_{\inf }^{\ln \langle|m|\rangle}$ & 7 & $0.988(20)$ & 0.43 \\
$\mathrm{~d} \ln \left\langle m^{2}\right\rangle / \mathrm{d} K$ at $K_{\inf }^{\ln \left\langle m^{2}\right\rangle}$ & 7 & $0.987(21)$ & 0.58 \\
Weighted average & & $0.986(14)$ & \\
$\mathrm{d} U / \mathrm{d} K$ at $K_{c}$ & 6 & $0.983(11)[71]$ & 0.64 \\
$\mathrm{~d} \ln \langle|m|\rangle / \mathrm{d} K$ at $K_{c}$ & 6 & $1.009(6)[44]$ & 0.42 \\
$\mathrm{~d} \ln \left\langle m^{2}\right\rangle / \mathrm{d} K$ at $K_{c}$ & 6 & $1.016(6)[38]$ & 0.23 \\
Weighted average & & $1.009(27)$ & \\
& & & \\
Overall average & & $0.991(12)$ & \\
\hline
\end{tabular}

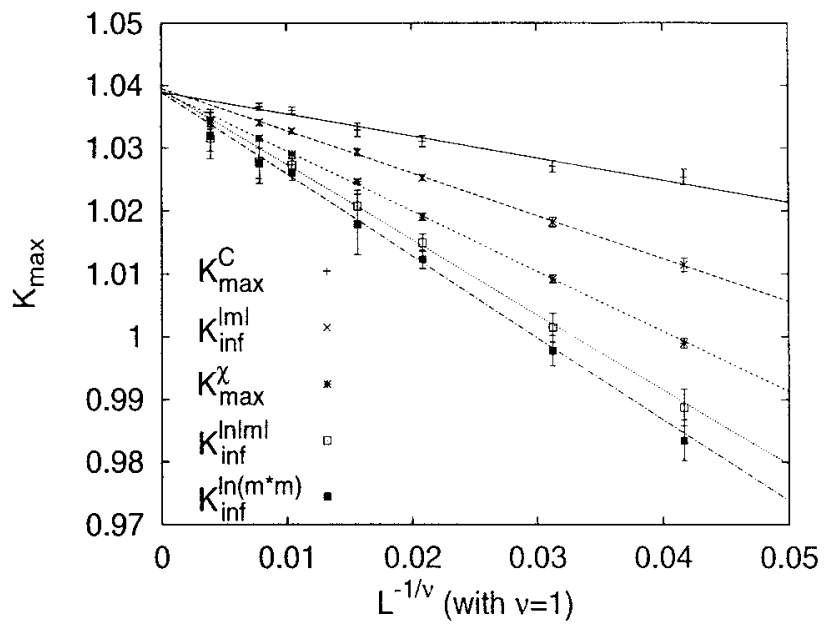

Fig. 1. Finite-size scaling extrapolations of pseudo-transition points $K_{\max }$ vs. $L^{-1 / v}$ for $a=0.001$, assuming $v=1$. The error-weighted average of extrapolations to infinite size yields $K_{c}=1.0393(3)$.

perfect agreement with the Onsager value $v=1$. For $a=0$ the scatter in the estimates is similar and the weighted average $1 / v=0.986(14)$ is again clearly compatible with $v=1$.

Assuming, therefore, $v=1$ we can obtain estimates for $K_{c}$ from linear least-squares fits to the scaling behavior of the various $K_{\max }$ sequences, as shown in Fig. 1 for $a=0.001$. Using again the fits with $L \geqslant 24$, the combined estimate from the five sequences leads to $K_{c}=1.0393$ (3) for $a=0.001$, and for $a=0$ with $L \geqslant 16$ we find $K_{c}=1.0395$ (3). These estimates are corroborated by the crossing point of the Binder cumulant curves, $K_{c}=1.03925(25)$ for $a=0.001$ and $K_{c}=1.0394(2)$ for $a=0$ (see Fig. 2). At the crossing point the cumulants take the (weakly) universal values of $U^{*}=0.612(1)$ $(a=0.001)$ and $U^{*}=0.612(4)(a=0)$. For $a=0.001$ we show in Fig. 3 the FSS 


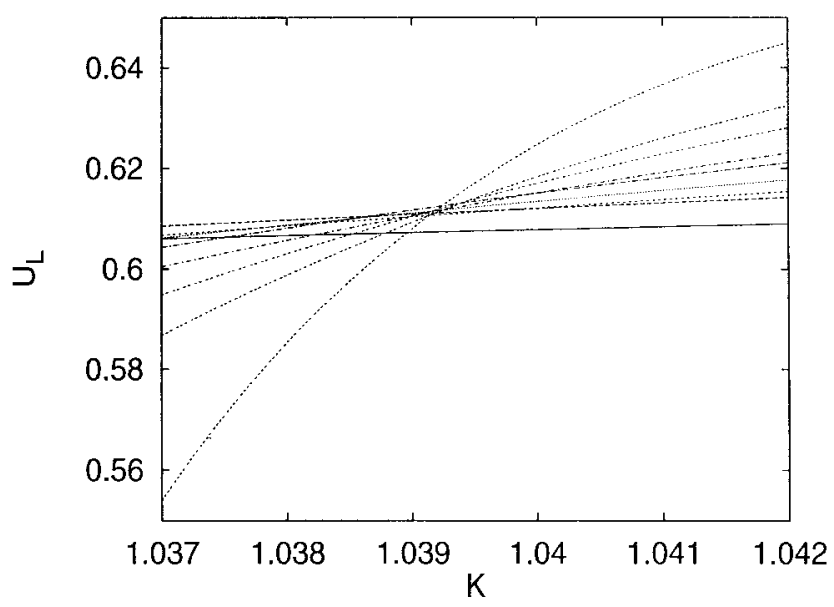

Fig. 2. Binder cumulant curves for $a=0.001$ leading to a crossing point at $K_{c}=1.03925(25)$ and $U_{L}=0.612(1)$.

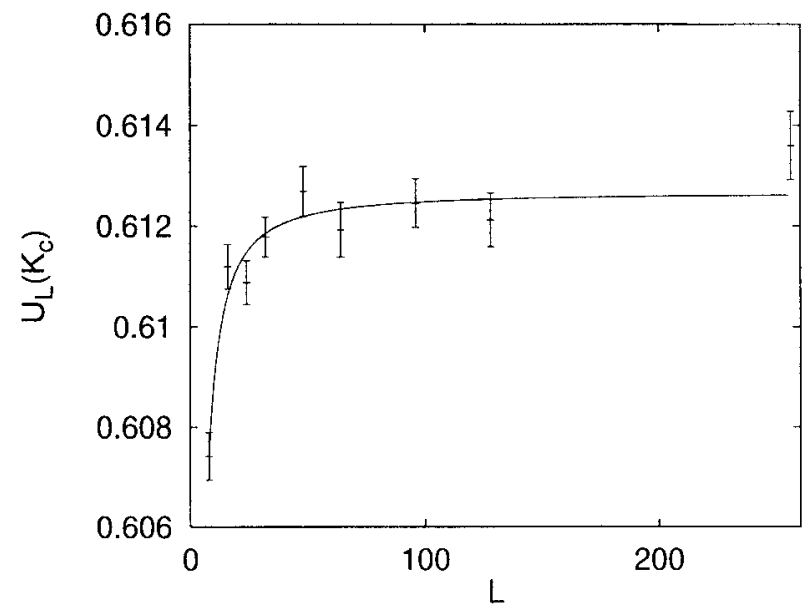

Fig. 3. Finite-size scaling of the Binder cumulant values $U_{L}(K)$ at $K=K_{c}=1.0393$ for $a=0.001$. The solid line is a three-parameter fit $U_{L}\left(K_{c}\right)=U^{*}+c L^{-w}$, yielding $U^{*}=0.6126(4)$ and $w=1.34(35)$.

behavior of $U_{L}$ at $K_{c}=1.0393$. The solid line is a three-parameter fit over all data using the ansatz $U_{L}\left(K_{c}\right)=U^{*}+c L^{-w}$ which yields $U^{*}=0.6126(4)$ and $w=1.34(35)$ with $Q=0.25$. These estimates are in full agreement with the study of the standard Regge calculus of Ref. [7] which gave $U^{*}=0.612(5)(a=0.001)$ and $U^{*}=0.609(33)(a=0)$. They are further compatible with the best available estimate of $U^{*}=0.6106901(5)$ in Ref. [22] for the regular 2D Ising model with periodic boundary conditions.

Knowing the critical coupling we may reconfirm our estimates of $1 / v$ by evaluating the above quantities at $K_{c}$. As can be inspected in Tables 1 and 2, the statistical errors of the FSS fits at $K_{c}$ are much smaller than those using the $K_{\max }$ sequences. However, here we also have to take into account the uncertainty in our estimate of $K_{c}$. This 


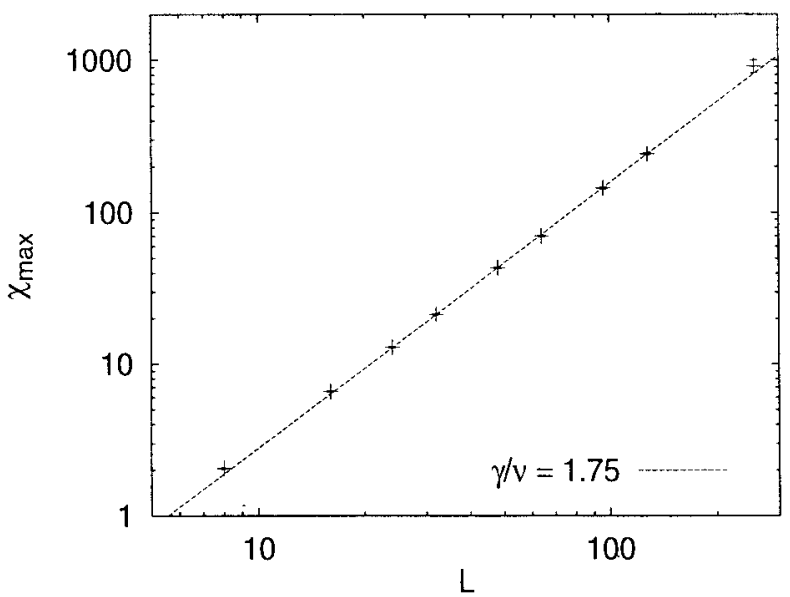

Fig. 4. Finite-size scaling of the susceptibility maxima $\chi_{\max }$ for $a=0.001$. The slope of the straight line is set to the Onsager value $\gamma / v=1.75$ for regular static lattices.

error is computed by repeating the fits at $K_{c} \pm \Delta K_{c}$ and indicated in Tables 1 and 2 by the numbers in square brackets. In the computation of the weighted average we assume the two types of errors to be independent. As a result of this combined analysis we obtain strong evidence that the exponent $v$ agrees with the standard Onsager value of $v=1$.

To extract the critical exponent ratio $\gamma / v(=2 \gamma / D v)$ we use the scaling $\chi_{\max } \cong L^{\gamma / v}$ as well as the scaling of $\chi$ at $K_{c}$, yielding for $a=0.001$ in the range $L=24-256$ estimates of $\gamma / v=1.741(11)$ with $Q=0.65$ and $\gamma / v=1.741(5)[22](Q=0.57)$, respectively. The corresponding values for $a=0$, using the range $L=16-128$, are $\gamma / v=1.747(8)(Q=0.60)$ and $\gamma / v=1.738(6)[11](Q=0.38)$. These estimates for $\gamma / v$ are fully consistent with the Onsager value of $\gamma / v=1.75$. In Fig. 4 this is demonstrated graphically for $a=0.001$ by comparing the scaling of $\chi_{\max }$ with a constrained one-parameter fit of the form $\chi_{\max }=c L^{1.75}$ with $c=0.0496(30)(Q=0.55, L \geqslant 24)$.

To extract the magnetic critical exponent ratio $\beta / v(=2 \beta / D v)$ we use that $\langle|m|\rangle \cong$ $L^{-\beta / v}$ at $K_{\text {inf }}^{|m|}$ and at $K_{c}$. Performing again fits in the range $L=24-256$ we obtain $\beta / v=0.118(12)(Q=0.70)$ and $\beta / v=0.127(1)[12](Q=0.31)$ for $a=0.001$ (cf. Fig. 5). For $a=0$ and a fit range of $L=16-128$ the corresponding results are $\beta / v=0.118(8)$ $(Q=0.74)$ and $\beta / v=0.125(1)[12](Q=0.15)$. Again the numerical estimates clearly agree within error bars with the Onsager value of $\beta / v=0.125$.

As a consistency check we have also analyzed the scaling behavior of the derivative $\mathrm{d}\langle|m|\rangle / \mathrm{d} K \cong L^{(1-\beta) / v}$. Here, we obtain for $a=0.001$ from the FSS fit with $L \geqslant 24$ at $K_{\text {inf }}^{|m|}$ an estimate of $(1-\beta) / v=0.874(12)(Q=0.46)$, and at $K_{c}$ we find $(1-\beta) / v=$ $0.885(5)[12](Q=0.19)$, to be compared with the Onsager value of $(1-\beta) / v=0.875$. For $a=0$ the corresponding fit results are $(1-\beta) / v=0.878(9)(Q=0.53, L \geqslant 16)$ and $(1-\beta) / v=0.884(6)[30](Q=0.48, L \geqslant 24)$, respectively. 


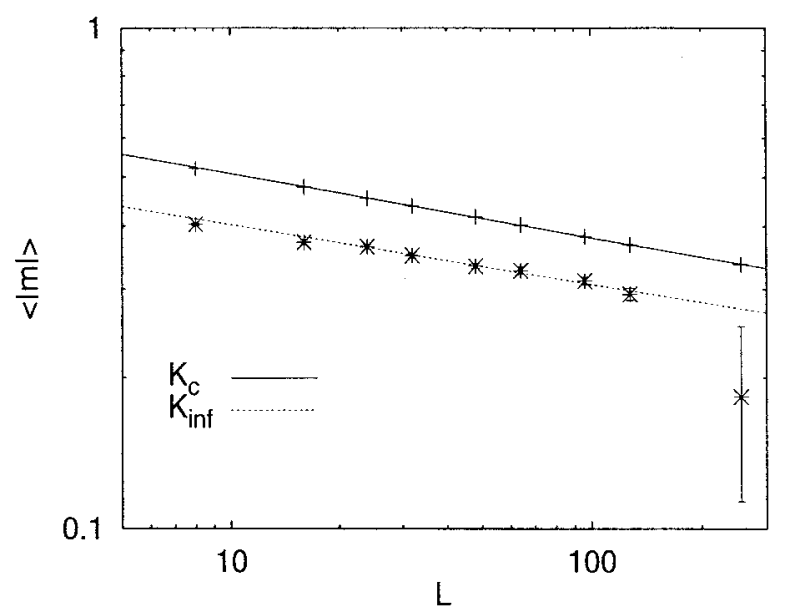

Fig. 5. Finite-size scaling of the magnetization $\langle|m|\rangle$ at $K_{c}=1.0393$ and at its points of inflection $K_{\text {inf }}^{|m|}(L)$ for $a=0.001$. The fits in the range $L=24-256$ yield $\beta / v=0.127(1)[12](Q=0.31)$ at $K_{c}$ and $\beta / v=0.118(12)$ $(Q=0.70)$ at $K_{\mathrm{inf}}^{|m|}(L)$.

Concerning the specific heat we expect in the case of the Onsager exponent $\alpha=0 \mathrm{a}$ logarithmic divergence of the form

$$
C(x, L)=A(x)+B(x) \ln L .
$$

Indeed, the data at the different fixed values of $x$ can all be fitted nicely with this ansatz. In particular, for the fit of $C_{\max }$ at $a=0.001$ with 7 points $(L \geqslant 24)$ we obtain $A=0.19(6), B=0.49(2)$, with a total $\chi^{2}=5.71$, and at $a=0$ with 7 data points $(L \geqslant 16) A=0.19(4), B=0.48(1)$, with a total $\chi^{2}=1.06$. We also tried an unbiased three-parameter fit using the power-law ansatz

$$
C(x, L)=A^{\prime}(x)+B^{\prime}(x) L^{\alpha / v},
$$

which gives us in the case of the fit of $C_{\max }$ for $a=0.001$ and 7 data points $A^{\prime} \approx$ $21.04, B^{\prime} \approx-20.94$, and $\alpha / v=-0.026(140)$, with a slightly improved total $\chi^{2}=5.68$ (cf. Fig. 6). It should be noted, however, that the three-parameter fit is highly unstable and exhibits strong correlations between the three parameters. This can be easily understood by expanding the power-law ansatz (3.2) for small $\alpha / v$ (and finite values of $L)$ as $C_{\max } \approx A^{\prime}+B^{\prime}+(\alpha / v) B^{\prime} \ln L+\cdots$. All fits with $A^{\prime}+B^{\prime}=0.10 \approx$ const. $\approx A$ and $(\alpha / v) B^{\prime}=0.54 \approx$ const. $\approx B$ are basically indistinguishable from the logarithmic ansatz (3.1). For $a=0$ and 7 data points we obtain $A^{\prime} \approx 30.34, B^{\prime} \approx-30.20$, and $\alpha / v=-0.017(78)$, with a total $\chi^{2}=1.02$. For comparison, we include in Fig. 6 for $a=0.001$ also the best linear least-squares fit with the KPZ prediction $\alpha / v=-2 / 3$ $(=2 \alpha / D v)$ of the form

$$
C(x, L)=A^{\prime \prime}(x)+B^{\prime \prime}(x) L^{-2 / 3},
$$

that renders for $C_{\max }$ the parameters $A^{\prime \prime}=2.82(3)$ and $B^{\prime \prime}=-9.3(3)$, with an unacceptably large total $\chi^{2}=25$. For $a=0$ we obtain with $A^{\prime \prime}=2.68(2)$ and $B^{\prime \prime}=-7.6(2)$ 


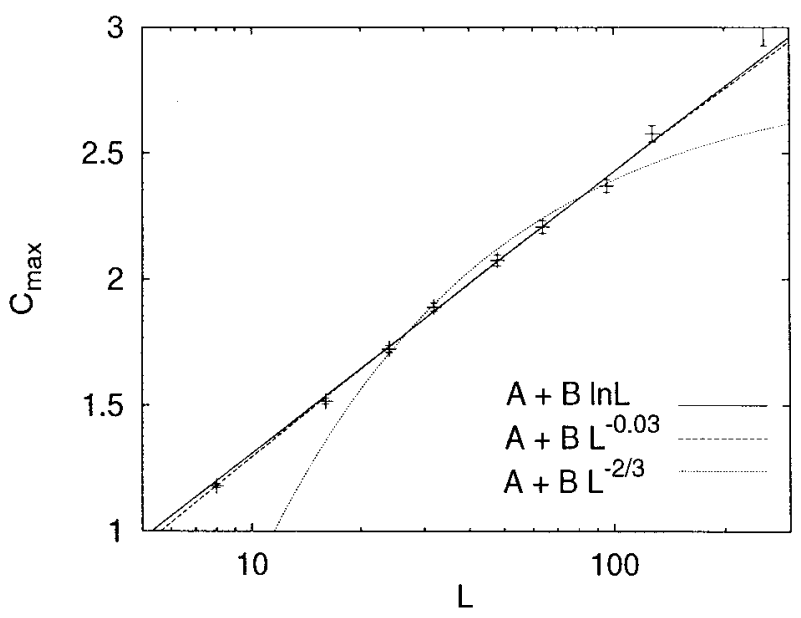

Fig. 6. Finite-size scaling of the specific-heat maxima $C_{\max }$ for $a=0.001$. A logarithmic fit $C_{\max }=A+B \ln L$, a power-law fit $C_{\max }=A+B L^{\alpha / v}$, and a constrained power-law fit assuming the KPZ prediction $\alpha / v=-\frac{2}{3}$ are shown.

an even larger chi-squared value of $\chi^{2}=66$. We can thus conclude that also for $\alpha / v$ our data is consistent with the flat-space Onsager value of zero.

\section{Concluding remarks}

We have performed a study of the Ising model coupled to quantum gravity via Regge calculus. Analyzing the discrete Regge model we have found that the critical exponents of the Ising transition still agree with the Onsager exponents for regular static lattices. Overall, we summarize that our results are fully consistent with the standard Regge calculus results [7]. The discrete Regge model can hence be used as a simplified approach for further investigations with non-local measures in the path integral.

\section{Acknowledgements}

E.B. thanks the Graduiertenkolleg "Quantenfeldtheorie: Mathematische Struktur und Anwendungen in der Elementarteilchen- und Festkörperphysik" for financial support during his extended stay in Leipzig.

\section{References}

[1] V.G. Knizhnik, A. Polyakov, A. Zamolodchikov, Mod. Phys. Lett. A 3 (1988) 819.

[2] J. Ambjørn, in: Proceedings of the 1994 Les Houches Summer School, Session LXII, hep-th/9411179.

[3] J. Ambjørn, Nucl. Phys. B 42 (Proc. Suppl.) (1995) 3.

[4] S. Catterall, Nucl. Phys. B 47 (Proc. Suppl.) (1996) 59. 
[5] D.A. Johnston, Nucl. Phys. B 53 (Proc. Suppl.) (1997) 43.

[6] M. Gross, H. Hamber, Nucl. Phys. B 364 (1991) 703.

[7] C. Holm, W. Janke, Phys. Lett. B 335 (1994) 143.

[8] C. Holm, W. Janke, Phys. Lett. B 375 (1996) 69.

[9] T. Regge, Nuovo Cimento 19 (1961) 558.

[10] P. Menotti, P. Peirano, Phys. Lett. B 353 (1995) 444.

[11] P. Menotti, P. Peirano, Nucl. Phys. B 473 (1996) 426.

[12] P. Menotti, P. Peirano, Nucl. Phys. B 488 (1997) 719.

[13] T. Fleming, M. Gross, R. Renken, Phys. Rev. D 50 (1994) 7363.

[14] W. Beirl, H. Markum, J. Riedler, Int. J. Mod. Phys. C 5 (1994) 359.

[15] W. Beirl, P. Homolka, B. Krishnan, H. Markum, J. Riedler, Nucl. Phys. B 42 (Proc. Suppl.) (1995) 710 .

[16] E. Bittner, A. Hauke, H. Markum, J. Riedler, C. Holm, W. Janke, Phys. Rev. D 59 (1999) 124018.

[17] W. Janke, Monte Carlo simulations of spin systems, in: K.H. Hoffmann, M. Schreiber (Eds.), Computational Physics: Selected Methods - Simple Exercises - Serious Applications, Springer, Berlin, 1996, p. 10.

[18] W. Janke, Nonlocal Monte Carlo Algorithms for Statistical Physics Applications, Math. Comput. Simulations 47 (1998) 329.

[19] U. Wolff, Phys. Rev. Lett. 62 (1989) 361.

[20] U. Wolff, Nucl. Phys. B 322 (1989) 759.

[21] A. Ferrenberg, R.H. Swendsen, Phys. Rev. Lett. 61 (1988) 2635; 63 (1989) 1658 (erratum).

[22] G. Kamieniarz, H.W.J. Blöte, J. Phys. A 26 (1993) 201. 